\title{
Compton Profiles for Atoms and Molecules: Compton Defects*
}

\author{
F. Gasser and M. Roeth
}

L.P.M.C., Institut de Physique et d'Électronique de Metz, Technopôle 2000, F-57070 Metz

Z. Naturforsch. 48a, 257-260 (1993); received December 24, 1991

A series expansion applied to the Born propagator present in the differential cross-section allows one to obtain successive corrections to the impulse approximation. The first and second corrections explain the essential features of the Compton defects. Results for the second corrective term are presented for $1 \mathrm{~s}, 2 \mathrm{~s}, 2 \mathrm{p}_{x, y}$, and $2 \mathrm{p}_{z}$ hydrogenic states. The decrease or increase of the scattered intensity near the maximum of the Compton profile is shown to be strongly related to geometric properties of the concerned orbital

Key words: Electronic structure; Ionisation cross-section; Compton scattering.

\section{Introduction}

In the impulse approximation (IA), Compton profiles are directly related to the electron momentum density or, by means of a Fourier transformation, to a position-space quantity, itself related to orbital autocorrelation functions [1]. Similarly, deviations from the IA that are present in experimental investigations of gaseous, solid and liquid systems, using electronimpact, X-ray or $\gamma$-ray techniques, can be interpreted in position space.

These deviations cause changes in the position of the Compton profile maximum and in the magnitude of the peak. In order to take these effects into account, a theoretical model has been proposed [2] and a first corrective term to the IA has been derived allowing an interpretation of the Compton shift. It is the aim of this paper to complete this study with the calculation of a second corrective term modifying the peak magnitude predicted by the IA.

\section{Theory}

In the first Born approximation (BA), the use of closure on the final states leads to an expression for the generalised Compton profile given by

$$
\begin{aligned}
J^{\mathrm{B}}(q, k)= & \frac{k}{2 \pi} \int_{-\infty}^{+\infty} \mathrm{e}^{-i t q k} \\
& \cdot \sum_{\mu=1}^{N}\left\langle\phi_{i}\left|\exp \left[i t\left(H-E_{i}-C_{\mu}\right)\right]\right| \phi_{i}\right\rangle \mathrm{d} t .
\end{aligned}
$$

\footnotetext{
* Presented at the Sagamore X Conference on Charge, Spin, and Momentum Densities, Konstanz, Fed. Rep. of Germany, September 1-7, 1991.
}

Reprint requests to Dr. F. Gasser, Laboratoire de Physique Moléculaire et des Collisions, Institut de Physique et d'Électronique de Metz, 1, Bd Arago, Technopôle 2000, F-57070 Metz, France.
$H$ represents the target Hamiltonian and $\phi_{i}$ the initialstate wave function of energy $E_{i}$. The usual Compton parameter $q$ is $\left(E-k^{2} / 2\right) / k$, where $E$ and $\boldsymbol{k}$ are the energy and momentum transferred from the incident particle to the target and $C_{\mu}=-i \boldsymbol{k} \nabla_{\mu}$. The Born operator, $\exp \left[i t\left(H-E_{i}-C_{\mu}\right)\right]$, is then expanded and $J^{\mathbf{B}}$ is written as a sum of terms alternatingly symmetric and antisymmetric,

$J^{\mathrm{B}}=J^{0}+J^{1}+J^{2}+\ldots+J^{n}+\ldots=J^{0}+J^{\mathrm{BA}}+J^{\mathrm{BS}}$.

$J^{0}(q)$ is the Compton profile in the IA. $J^{\mathrm{BA}}(q, k)$ and $J^{\mathrm{BS}}(q, k)$ represent the sums of all the corrective terms to $J^{0}$, antisymmetric and symmetric, respectively. An approximate expression for $J^{\mathbf{B}}$ is introduced with

$$
J=J^{0}+J^{1}+J^{2} .
$$

For hydrogenic ions with nuclear charge $Z, J^{\mathrm{B}}$ is well known [3], and the first corrective term $J^{1}$ can be compared to $J^{\mathrm{BA}}$ and $J^{2}$ to $J^{\mathrm{BS}}$. In this simple case, the validity of the proposed approximation can be estimated. $J^{0}, J^{1}$, and $J^{2}$ can be written as

$$
J^{0}(q)=\frac{1}{\zeta \pi} \int_{0}^{\infty} \cos (Q X) S(X) \mathrm{d} X
$$
with $S(X)=\left\langle\phi\left(\boldsymbol{r}_{b}\right) \mid \phi\left(\boldsymbol{r}_{a}\right)\right\rangle, Q=\frac{q}{\zeta}$ and $X=\zeta k t$ ( $\zeta$ is
the orbital exponent);

$$
\begin{aligned}
& J^{1}(q, k)=-\frac{1}{\zeta \pi} \frac{Z}{k} \int_{0}^{\infty} \sin (Q X) \mathrm{d} X \\
& \cdot \int_{0}^{X} \frac{1}{\zeta}\left\langle\phi_{i}\left(r_{b}\right)\left|\frac{1}{r_{c}}-\frac{1}{r_{b}}\right| \phi_{i}\left(r_{a}\right)\right\rangle \mathrm{d} X_{1}, \\
& J^{2}(q, k)=-\frac{1}{\zeta \pi}\left(\frac{Z}{k}\right)^{2} \int_{0}^{\infty} \cos (Q X) \mathrm{d} X \\
& \cdot \int_{0}^{X} \int_{0}^{X_{1}} \frac{1}{\zeta^{2}}\left\langle\phi_{i}\left(r_{b}\right)\left|\left(\frac{1}{r_{c}}-\frac{1}{r_{b}}\right)\left(\frac{1}{r_{d}}-\frac{1}{r_{a}}\right)\right| \phi_{i}\left(r_{a}\right)\right\rangle \mathrm{d} X_{2} \mathrm{~d} X_{1},
\end{aligned}
$$

0932-0784 / $93 / 0100-0257 \$ 01.30 / 0$. - Please order a reprint rather than making your own copy. 
The parameters used in the integrations are represented in Fig. 1 and fulfil the relationships

$\boldsymbol{R}=\boldsymbol{k} t, \quad \boldsymbol{R}_{1}=\boldsymbol{k} t_{1}, \quad \boldsymbol{R}_{2}=\boldsymbol{k} t_{2}$,

$r_{a}=r, \quad r_{b}=r-R, \quad r_{c}=r-R_{1}, \quad r_{d}=r-R_{2}$.

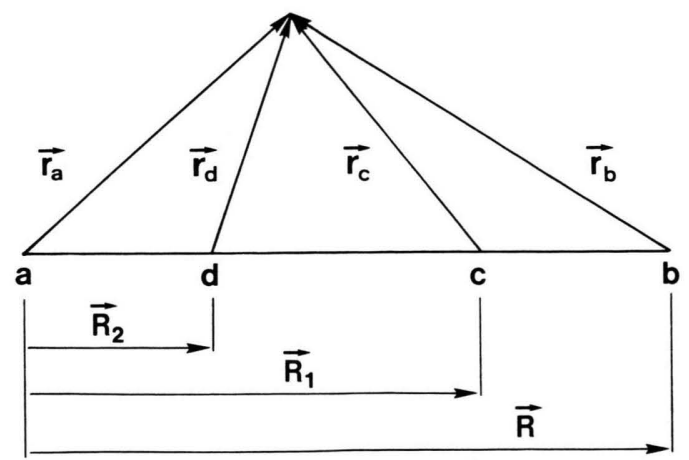

Fig. 1. Geometrical representation of parameters used in (6).
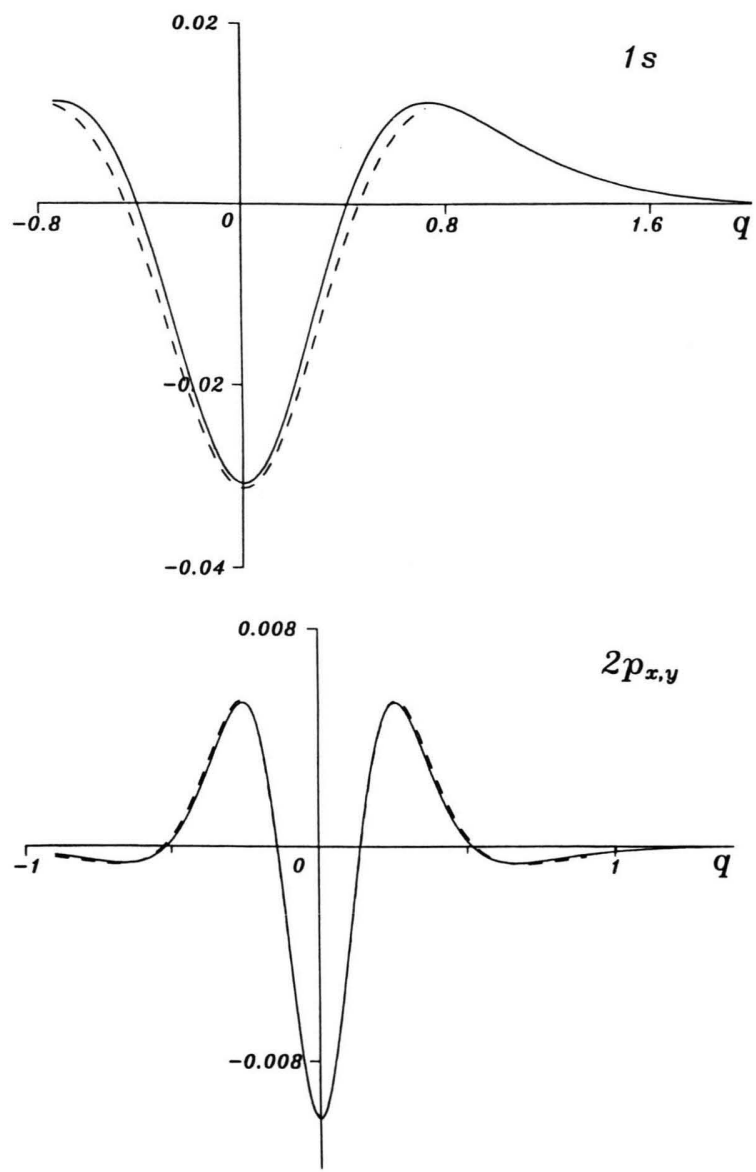

Fig. 2. The second correction $J^{2}(-)$ compared to the sum of all the symmetric corrections $J^{\mathrm{BS}}(--)$ to the impulse approximation ( $k=2$ a.u., $Z=1$ ).
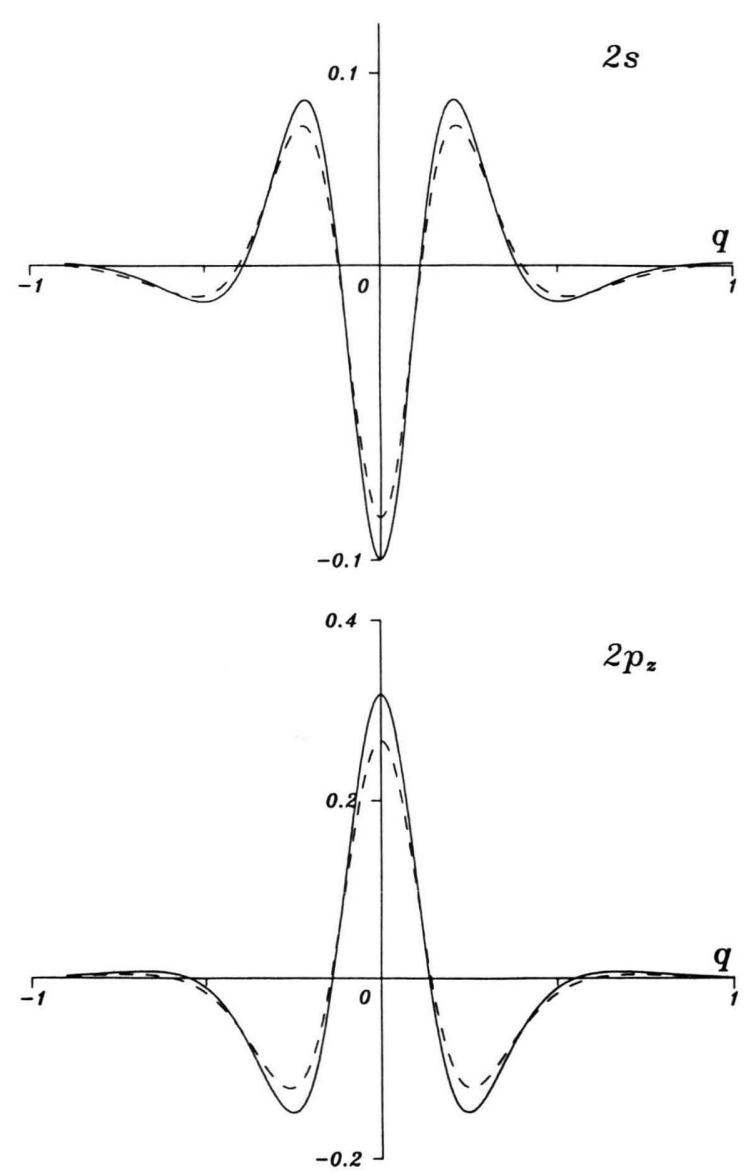

The general term in the expansion (2) may be expressed as

$$
J^{n}=\frac{1}{\zeta}\left(\frac{Z}{k}\right)^{n} j^{n}(Q)
$$

$j^{n}(Q)$ is a function characteristic of the orbital from which the electron is ejected. $J^{1}$ and $J^{2}$ are multicentre potential-energy integrals including binding effects due to the nuclear charge $Z$ acting upon the ejected electron during its motion, which is assumed to be linear. For a given hydrogenic state, if $Z / k$ is sufficiently small, the expansion (2) converges rapidly, $J^{1}$ and $J^{2}$ represent a good approximation to $J^{\mathrm{BA}}$ and $J^{\mathrm{BS}}$, respectively, and $\Delta J=J^{1}+J^{2}$ furnishes a good estimate of the total defect over the whole experimental profile after subtraction of $J^{0} . J^{1}$, which has been extensively studied [2-4], is mainly responsible for the shift of the Compton peak and allows only compari- 

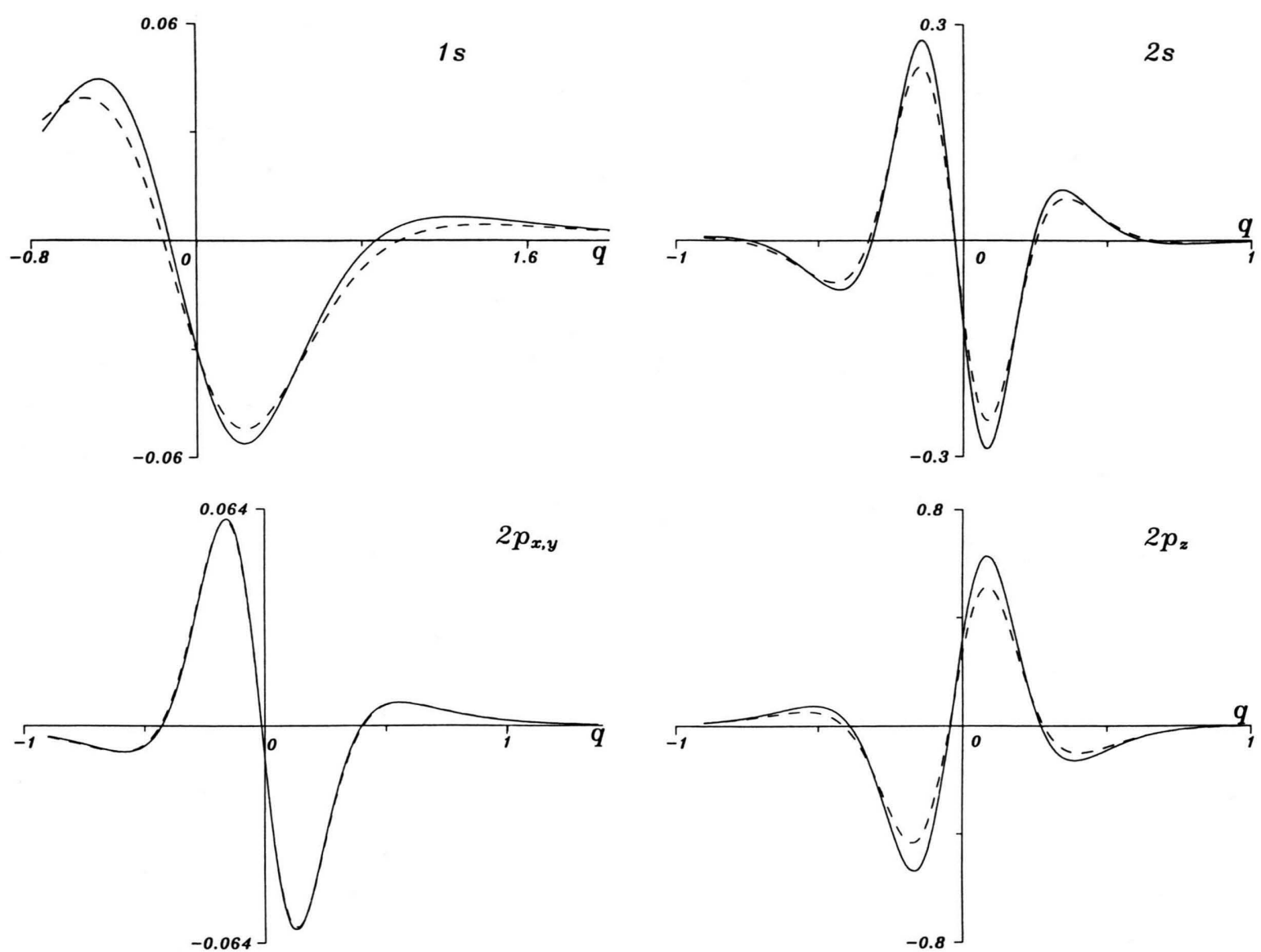

Fig. 3. The total Compton defect calculated with the proposed approximation $\Delta J(-)$ and with the Born approximation $(--)(k=2$ a.u., $Z=1)$.

son with the antisymmetrical part of the experimental profile over a symmetric range in $q$.

\section{Results and Discussion}

The results are presented in Figs. 2, 3, and 4 for the hydrogenic states $1 \mathrm{~s}, 2 \mathrm{~s}, 2 \mathrm{p}_{x, y}$, and $2 \mathrm{p}_{z}$ (orbital axis perpendicular and parallel to the direction of $\boldsymbol{k}$ for $2 \mathrm{p}_{x, z}$ and $2 \mathrm{p}_{z}$, respectively). The calculations have been made for $k=2$ a.u. and for a nuclear charge $Z=1$ so that $Z / k=1 / 2$ whatever the state is, but the average energy transferred to the target is 4 times the binding energy for a $1 \mathrm{~s}$ electronic state and 16 times for an electron ejected from the L-shell. Simple analytical formulas are obtained for $q=0$ where $J^{1}=0$. They are presented in Table 1 together with numerical results illustrating the convergence of the expansion (2) by $J^{2} / J^{0}$, the validity of the IA by $\left(J^{\mathrm{B}}-J^{0}\right) / J^{\mathrm{B}}$ and the validity of the proposed treatment by $\left(J^{\mathrm{B}}-J\right) / J^{\mathrm{B}}$.
The differences in the behaviour of $J^{2}$, depending on the nature of the concerned orbital, are similar to those of $J^{1}$ and can be explained in the same manner [2]. For the hydrogenic states $2 \mathrm{~s}, 2 \mathrm{p}_{x, y}$ and $2 \mathrm{p}_{z}$ with the same binding energy, the larger values of $J^{2}$ and $J^{2} / J^{0}$ are observed for these orbitals, which give rise to the larger spatial extent of the self-overlap function $S(R)$ in the $\boldsymbol{k}$ direction (Figure 5). Moreover, for the $2 \mathrm{p}_{z}$ state, the negative part of the overlap function occurs at large $R$ values and leads to a positive sign for $J^{2}$ in the neighbourhood of $q=0$, increasing the Compton peak height. When $k / Z$ is sufficiently large, $\left(J^{\mathrm{B}}-J^{0}\right) / J^{\mathrm{B}}$ behaves as $Z^{2} / k^{2}$, like $J^{2} / J^{0}$ (this can be noticed in the Table). In that case, for a given orbital, the Compton defect increases with increasing $Z$ and the binding energy effects are dominant. In all other cases there is competition between energetic and geometric properties of the orbitals.

Finally, it can be remarked that the agreement between the proposed model and the BA increases when 
Table 1. Calculated values of the symmetric correction to the impulse approximation at $q=0$ for $k=2$ a.u. and $Z=1\left(2 \mathrm{p}_{\mathrm{av}}\right.$ means the averaged $2 \mathrm{p}_{x, y}, 2 \mathrm{p}_{z}$ values).

\begin{tabular}{llllll}
\hline & $1 \mathrm{~s}$ & $2 \mathrm{~s}$ & $2 \mathrm{p}_{x, y}$ & $2 \mathrm{p}_{z}$ & $2 \mathrm{p}_{\mathrm{av}}$ \\
\hline$J^{2}$ & $\frac{Z^{2}}{k^{2} \zeta} \frac{4\left(9-\pi^{2}\right)}{9 \pi}$ & $\frac{Z^{2}}{k^{2} \zeta} \frac{2\left(141-16 \pi^{2}\right)}{45 \pi}$ & $\frac{Z^{2}}{k^{2} \zeta} \frac{2\left(39-4 \pi^{2}\right)}{15 \pi}$ & $\frac{Z^{2}}{k^{2} \zeta} \frac{2}{\pi}$ & $\frac{Z^{2}}{k^{2} \zeta} \frac{2\left(93-8 \pi^{2}\right)}{45 \pi}$ \\
\hline$J^{2} / J^{0}$ & $\frac{Z^{2}}{k^{2}} \frac{\left(9-\pi^{2}\right)}{6}$ & $\frac{Z^{2}}{k^{2}} \frac{\left(141-16 \pi^{2}\right)}{96}$ & $\frac{Z^{2}}{k^{2}} \frac{\left(39-4 \pi^{2}\right)}{24}$ & - & $\frac{Z^{2}}{k^{2}} \frac{\left(93-8 \pi^{2}\right)}{48}$ \\
\hline$J^{2}$ & -0.03 & -0.12 & -0.01 & 0.32 & 0.10 \\
\hline$J^{2} / J^{0}$ & $-3.6 \%$ & $-4.4 \%$ & $-0.5 \%$ & - & $7.3 \%$ \\
\hline$\left(J^{\mathrm{B}}-J^{0}\right) / J^{\mathrm{B}}$ & $-3.8 \%$ & $-3.9 \%$ & $-0.5 \%$ & $100 \%$ & $5.7 \%$ \\
\hline$\left(J^{\mathrm{B}}-J\right) / J^{\mathrm{B}}$ & $-0.07 \%$ & $0.66 \%$ & $5.10^{-4} \%$ & $-19 \%$ & $-1.2 \%$ \\
\hline
\end{tabular}
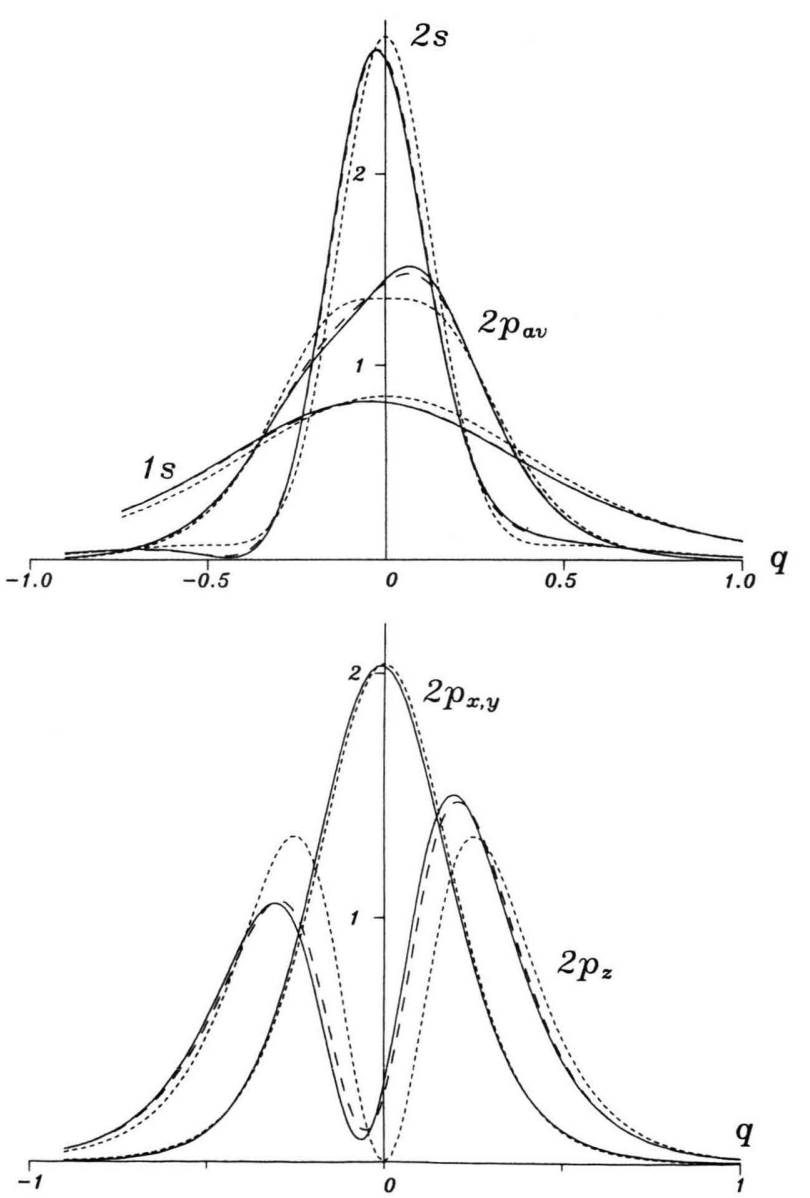

Fig. 4. Compton profiles represented in the impulse approximation $(\cdots)$, the Born approximation $(--)$ and the proposed approximation $(\longrightarrow) .(k=2$ a.u., $Z=1),\left(2 \mathrm{p}_{\mathrm{av}}\right.$ means the averaged $2 \mathrm{p}_{x, y}, 2 \mathrm{p}_{z}$ values).

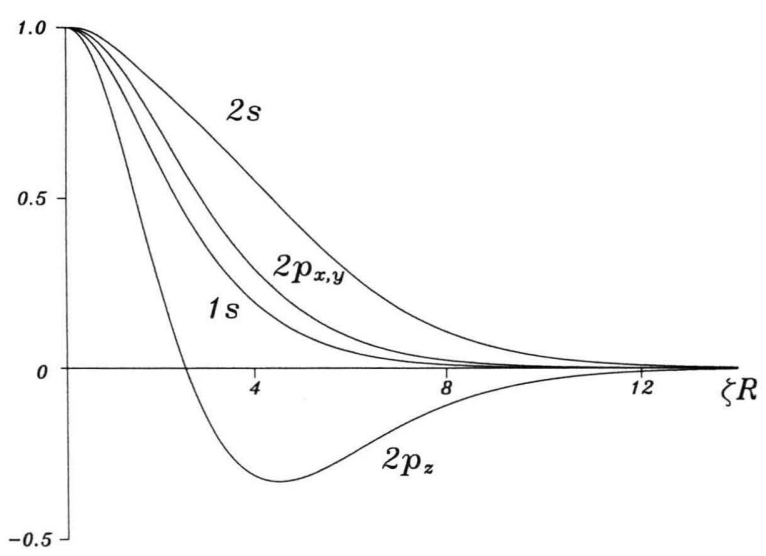

Fig. 5. Self-overlap functions (reciprocal form factors [1]) for $1 \mathrm{~s}, 2 \mathrm{~s}$, and $2 \mathrm{p}$ hydrogenic orbitals.

$J^{2} / J^{0}$ decreases. So the calculation of $J^{2} / J^{0}$ at $q=0$ allows to estimate the validity of the IA and to test the convergence of the proposed expansion of $J^{\mathrm{B}}$. For complex systems like atoms and molecules, $J^{2}$ at $q=0$ is the sum of the contributions of the individual orbitals with an effective nuclear charge extracted from $J^{1}$ at $q=0$.

[1] W. Weyrich, P. Pattison, and B. G. Williams, Chem. Phys. 41, 271 (1979).

[2] F. Gasser and C. Tavard, Phys. Rev. A 27, 117 (1983).

[3] B. J. Bloch and L. B. Mendelsohn, Phys. Rev. A 9, 129 (1974).

[4] C. Tavard, M. C. Dal Cappello, F. Gasser, and C. Dal Cappello, Phys. Rev. A 27, 199 (1983). 